\title{
Про структуру матриць над областю головних ідеалів відносно перетворення подібності
}

\author{
Володимир Прокіп
}

\begin{abstract}
The structure of matrices over a domain of principal ideals with respect to similarity transformations is investigated. The article consists of four chapters. In the second section supporting results are presented. In particular, we construct the triangular form of the matrix with respect to the similarity transformation for the case when its minimal polynomial decomposes into the product of different linear factors. In Section 3 it is proved that the Hessenberg form of a matrix $A$ with an irreducible minimal quadratic polynomial $m(\lambda)$ is a block triangular matrix with blocks of dimensional $2 \times 2$ with characteristic polynomials $m(\lambda)$ on the main diagonal. In the fourth section it is proved that a matrix $A$ with the minimal polynomial $m(\lambda)=(\lambda-\alpha)(\lambda-\beta), \alpha \neq \beta$, is similar to the lower block-triangular matrix, the diagonal blocks of which are diagonal matrices with elements $\alpha$ i $\beta$ on the main diagonals respectively. As a result of this the canonical form of involutory matrices over the ring of integers with respect to similarity transformations is established.
\end{abstract}

Анотація. В статті досліджується структура матриць над областю головних ідеалів відносно перетворення подібності. В другому розділі наведено допоміжні результати. В цьому розділі вказано трикутну форму матриці відносно перетворення подібності, мінімальний многочлен якої розкладається в добуток різних лінійних множників. В розділі 3 доведено, що форма Хессенберга матриці $A$ з незвідним мінімальним квадратичним многочленом $m(\lambda)$ є блочно-трикутна матриця з блоками вимірності $2 \times 2$ на головній діагоналі та з характеристичними многочленами $m(\lambda)$. У четвертому розділі доведено, що матриця $A$ із мінімальним многочленом $m(\lambda)=(\lambda-\alpha)(\lambda-\beta), \alpha \neq \beta$ подібна нижній блочно-трикутній матриці, діагональними блоками якої є діагональні матриці з елементами $\alpha$ і $\beta$ на головних діагоналях відповідно. Як наслідок вказано канонічну форму інволютивної матриці над кільцем цілих чисел відносно перетворень подібності.

2010 Mathematics Subject Classification: 15A04, 15A21, 15B36, 11C20

УДК 512.643

Ключові слова: область головних ідеалів, матриця, подібність 


\section{1. ВСТУП}

Нехай $\mathrm{R}$ - область головних ідеалів з одиницею $e \neq 0$. Позначимо: $M_{m, n}(\mathrm{R})$ - множина $(m \times n)$-матриць над $\mathrm{R} ; I_{n}$ - одинична $n \times n$ матриця; $0_{n, m}$ - нульова $n \times m$ матриця і $\mathrm{O}-$ нульова матриця, вимірність якої визначатиметься з контексту. Надалі через $a(\lambda)$ позначатимемо характеристичний многочлен матриці $A \in M_{n, n}(\mathrm{R})$, тобто $a(\lambda)=\operatorname{det}\left(I_{n} \lambda-A\right)$, а через $m(\lambda)$ ї̈ мінімальний многочлен.

Кажуть, що матриці $A, B \in M_{n, n}(\mathrm{R})$ подібні, якщо існує матриця $T \in \mathrm{GL}(n, \mathrm{~K})$ така, що $T A T^{-1}=B$. Структура матриць над полем відносно перетворень подібності описана повністю, див. [16, 3, 6, 12]. Проте задача про описання структури матриць відносно перетворень подібності при переході до довільного комутативного кільця з одиницею значно ускладнюється. В більшості випадків ця задача включає в себе класичну нерозв'язну задачу про канонічну форму пари матриць над полем відносно перетворення подібності, $[18,15,26]$. На даний час такі задачі називають дикими, [19, 20].

Дослідження подібності матриць над кільцями були і є в центрі уваги багатьох математиків. Можна стверджувати, що задача про подібність матриць над кільцем цілих чисел вперше досліджувалась в статті K. Латімера і К. К. Макдаффі [10]. Починаючи з 60-х років минулого століття задача про подібність матриць над комутативними кільцями досліджується в роботах як вітчизняних так і закордонних математиків. Серед них відзначимо роботи О. Таусскі [13], Д. А. Супруненка [26], В. Р. Макдональда [11], А. А. Нечаєва [21], Р. М. Гуральника [7] та ін.

На даний час теорія про описання структури матриць над комутативними кільцями, які не є полем, відносно перетворень подібності далека до свого завершення. Більшість досліджень, які стосуються задачі про подібність матриць над кільцями, розглядалися при тих чи інших обмеженнях на кільце $[5,9,11]$, на порядок матриці $[17,25,1,4]$, або на її характеристичний многочлен [22, 24]. Огляд окремих результатів, які стосуються подібності матриць над комутативними кільцями наведено в монографіях $[3,6,12]$. Проте канонічних форм матриць щодо перетворення подібності в цих роботах не встановлено.

В роботі [22] вказано умови, за яких матриця над областю головних ідеалів з мінімальним квадратичним многочленом перетвореннями подібності приводяться до діагонального вигляду. Дана робота є продовженням цих досліджень. В другому розділі наведено допоміжні результати. Зокрема, в твердженні 2.3 вказано трикутну форму для матриць, мінімальний многочлен яких розкладається в добуток лінійних множників і немає кратних коренів, відносно перетворення подібності. В розділі 3 доведено, що форма Хессенберга матриці $A$ з незвідним 
мінімальним многочленом $m(\lambda)=\lambda^{2}+m_{1} \lambda+m_{2}$ є блочно-трикутна матриця з блоками вимірності $2 \times 2$ на головній діагоналі та 3 характеристичними многочленами $m(\lambda)$ (Теорема 3.1 ). У четвертому розділі досліджується структура матриць із мінімальним квадратичним многочленом $m(\lambda)=(\lambda-\alpha)(\lambda-\beta), \alpha \neq \beta$. Основним результатом даної статті є теорема 4.1. Вона встановлює, що матриця $A$ із мінімальним многочленом $m(\lambda)$ подібна нижній блочно-трикутній матриці, блоками якої є діагональні матриці. Як наслідок отримано, що для інволютивної матриці над кільцем цілих чисел така трикутна матриця єдина.

\section{2. ДОПОМІЖні ТВЕРДЖЕННЯ}

В цій частині наведемо твердження, які будуть використані при доведенні основних результатів цієї статті.

Зауваження 2.1. Нехай $m(\lambda)=\lambda^{k}+m_{1} \lambda^{k-1}+\cdots+m_{k} \in \mathrm{R}[\lambda]-$ мінімальний многочлен матриці

$$
A=\left[\begin{array}{cc}
A_{1} & 0_{r, n-r} \\
A_{21} & A_{2}
\end{array}\right] \in M_{n, n}(\mathrm{R})
$$

де $A_{1} \in M_{r, r}(\mathrm{R}), r<n$. Тоді $m(\lambda)$ анулюючий многочлен матриць $A_{1} \mathrm{i}$ $A_{2}$.

Доведення. Так як $A^{0}=I_{n}$ i $A^{i}=\left[\begin{array}{cc}A_{1}^{i} & 0_{r, n-r} \\ * & A_{2}^{i}\end{array}\right]$ для всіх $i=1,2, \ldots$, TO

$$
m(A)=m\left(\left[\begin{array}{cc}
A_{1} & 0_{r, n-r} \\
* & A_{2}
\end{array}\right]\right)=0_{n, n} .
$$

Звідси отримуємо $m\left(A_{1}\right)=0_{r, r}$ і $m\left(A_{2}\right)=0_{n-r, n-r}$, що і доводить зауваження.

Твердження 2.2. Якщо характеристичний многочлен матриці $A \in$ $M_{n, n}(\mathrm{R})$ допускае зображення у вигляді добутку $а(\lambda)=(\lambda-\alpha) b(\lambda)$, де $\alpha \in \mathrm{R}$, то для А існуе матриия $U \in \mathrm{GL}(n, \mathrm{R})$ така, що

$$
U A U^{-1}=\left[\begin{array}{cccc}
\alpha & 0 & \ldots & 0 \\
A_{21} & & A_{22} &
\end{array}\right], \quad \text { de } \quad A_{22} \in M_{n-1, n-1}(\mathrm{R}) .
$$

Доведення. Оскільки $I_{n} \alpha-A$ особлива матриця, то для $A$ існує власний вектор $\bar{u} \in M_{1, n}(\mathrm{R})$, який відповідає власному значенню $\alpha$, тобто $\bar{u} A=\bar{u} \alpha$. Серед власних лівих векторів, які відповідають власному значенню $\alpha$, існує вектор $\bar{u}_{0}=\left[\begin{array}{llll}u_{01} & u_{02} & \ldots & u_{0 n}\end{array}\right] \in M_{1, n}(\mathrm{R})$ такий, що елементи $\left\{u_{01}, u_{02}, \ldots, u_{0 n}\right\}$ взаємно прості. Вектор $\bar{u}_{0}$ доповнюється до оборотної матриці, див. [12, Corollary II.1], тобто існує матриця 
$U \in \mathrm{GL}(n, \mathrm{R})$, першим рядком якої є вектор $\bar{u}_{0}$. Так як $U A=\left[\begin{array}{c}\alpha \bar{u}_{0} \\ *\end{array}\right]$, то $U A U^{-1}=\left[\begin{array}{cccc}\alpha & 0 & \ldots & 0 \\ A_{21} & & A_{22} & \end{array}\right]$. Твердження доведено.

Твердження 2.3. Нехай характеристичний многочлен матриці $A \in$ $M_{n, n}(\mathrm{R})$ допускає зображення у вигляді добутку

$$
a(\lambda)=\left(\lambda-\alpha_{1}\right)^{k_{1}}\left(\lambda-\alpha_{2}\right)^{k_{2}} \cdots\left(\lambda-\alpha_{r}\right)^{k_{r}},
$$

де $\alpha_{i} \in \mathrm{R} i k_{i} \geq 1$ для всіх $i=1,2, \ldots, r$. Якщо мінімальний многочлен матриці $А$ не мае кратних коренів, тобто

$$
m(\lambda)=\left(\lambda-\alpha_{1}\right)\left(\lambda-\alpha_{2}\right) \cdots\left(\lambda-\alpha_{r}\right)
$$

то для $A$ існуе матриия $U \in \mathrm{GL}(n, \mathrm{R})$ така, що

$$
U A U^{-1}=\left[\begin{array}{ccccc}
\alpha_{1} I_{k_{1}} & \mathrm{O} & \ldots & \ldots & \mathrm{O} \\
A_{21} & \alpha_{2} I_{k_{2}} & \mathrm{O} & \ldots & \mathrm{O} \\
\ldots & \ldots & \ldots & \ldots & \ldots \\
A_{r 1} & A_{r 2} & \ldots & A_{r, r-1} & \alpha_{r} I_{k_{r}}
\end{array}\right]
$$

Доведення. Нехай характеристичний многочлен матриці $A \in M_{n, n}(\mathrm{R})$ зображений у вигляді добутку (2.1). Згідно твердження 2.2 отримуємо, що для $A$ існує матриця $U \in \mathrm{GL}(n, \mathrm{R})$ така, що

$$
U A U^{-1}=\left[\begin{array}{ccccc}
A_{1} & \mathrm{O} & \ldots & \ldots & \mathrm{O} \\
A_{21} & A_{2} & \mathrm{O} & \ldots & \mathrm{O} \\
\ldots & \ldots & \ldots & \ldots & \ldots \\
A_{r 1} & A_{r 2} & \ldots & A_{r, r-1} & A_{r}
\end{array}\right]
$$

де $A_{i} \in M_{k_{i}, k_{i}}(\mathrm{R})=\left[\begin{array}{ccccc}\alpha_{i} & 0 & \ldots & \ldots & 0 \\ \alpha_{21}^{i} & \alpha_{i} & 0 & \ldots & 0 \\ \ldots & \ldots & \ldots & \ldots & \ldots \\ \alpha_{k_{1}, 1}^{i} & \alpha_{k_{1}, 2}^{i} & \ldots & \alpha_{k_{1}, k_{1}-1}^{i} & \alpha_{i}\end{array}\right] \in M_{k_{i}, k_{i}}(\mathrm{R})-$ нижні трикутні матриці з елементами $\alpha_{i}$ на головних діагоналях для всіх $i=1,2, \ldots, r$. На підставі зауваження 2.1 отримуємо $m\left(A_{i}\right)=0_{k_{i}, k_{i}}$. Останню рівність запишемо в розгорнутому вигляді

$$
\begin{gathered}
m\left(A_{i}\right)=\left(\alpha_{1} I_{k_{i}}-A_{i}\right) \cdots \\
\cdots\left(\alpha_{i-1} I_{k_{i}}-A_{i}\right)\left(\alpha_{i} I_{k_{i}}-A_{i}\right)\left(\alpha_{i+1} I_{k_{i}}-A_{i}\right) \cdots \\
\cdots\left(\alpha_{r} I_{k_{i}}-A_{i}\right)=0_{k_{i}, k_{i}}
\end{gathered}
$$

для всіх $i=1, \ldots, r$. 
Так як $A_{i} \in M_{k_{i}, k_{i}}(\mathrm{R})$ - нижні трикутні матриці, то

$$
A_{i}-\alpha_{j} I_{k_{i}}=\left[\begin{array}{llllc}
\alpha_{i}-\alpha_{j} & 0 & 0 & \ldots & 0 \\
\alpha_{21}^{i} & \alpha_{i}-\alpha_{j} & 0 & \ldots & 0 \\
\ldots & \ldots & \ldots & \ldots & \ldots \\
\alpha_{k_{i}-1, k_{i}}^{i} & \ldots & \alpha_{k_{i}-1, k_{i}-2}^{i} & \alpha_{i}-\alpha_{j} & 0 \\
\alpha_{k_{1}, 1}^{i} & \ldots & \alpha_{k_{i}, k_{1}-2}^{i} & \alpha_{k_{i}, k_{1}-1}^{i} & \alpha_{i}-\alpha_{j}
\end{array}\right]
$$

- нижні трикутні матриці з елементами $\alpha_{i}-\alpha_{j}$ на головних діагоналях для всіх $i, j=1,2, \ldots, r$. Очевидно, що для всіх $i \neq j$ матриці $\left(\alpha_{j} I_{k_{i}}-A_{i}\right)$ є неособливими і $\left(\alpha_{i} I_{k_{i}}-A_{i}\right)$ - особлива матриця. На підставі сказаного з рівності (2.2) випливає $A_{i}=\alpha_{i} I_{k_{i}}$. Так як $1 \leq i \leq r$, то $A_{i}=\alpha_{i} I_{k_{i}}$ для всіх $i=1,2, \ldots, r$. Твердження доведено.

Із твердження 2.3 отримуємо достатню умову діагоналізовності матриць над областю головних ідеалів (див. [23], наслідок 1) та альтернативне доведення відомого критерію діагоналізовності матриць над полем.

Нехай $A \in M_{m, m}(\mathrm{R}), B \in M_{n, n}(\mathrm{R}), C_{1}, C_{2} \in M_{n, m}(\mathrm{R})$. Відомо, [8], що матричне рівняння $B X-X A=C_{1}$ сумісне тоді і тільки тоді, коли матриці

$$
M_{0}=\left[\begin{array}{cc}
A & 0_{m, n} \\
0_{n, m} & B
\end{array}\right] \quad \text { i } \quad M_{1}=\left[\begin{array}{cc}
A & 0_{m, n} \\
C_{1} & B
\end{array}\right]
$$

подібні. Отже, серед матриць $T \in \mathrm{GL}(m+n, \mathrm{R})$ таких, що $T M_{0}=M_{1} T$ існує нижня трикутна матриця $T_{0}=\left[\begin{array}{cc}T_{1} & 0_{m, n} \\ T_{21} & T_{2}\end{array}\right]$, де $T_{1} \in \mathrm{GL}(m, \mathrm{R})$ i $T_{2} \in \mathrm{GL}(n, \mathrm{R})$, така, що $T_{0} M_{0}=M_{1} T_{0}$.

B [14] поставлено наступну гіпотезу. Припустимо, що матриці

$$
M_{1}=\left[\begin{array}{cc}
A & 0_{m, n} \\
C_{1} & B
\end{array}\right] \quad \text { i } \quad M_{2}=\left[\begin{array}{cc}
A & 0_{m, n} \\
C_{2} & B
\end{array}\right]
$$

подібні. Тоді серед зворотних матриць $T \in \mathrm{GL}(m+n, \mathrm{R})$ існує нижня блочно-трикутна матриця $T_{0}=\left[\begin{array}{cc}T_{11} & 0_{m, n} \\ T_{21} & T_{22}\end{array}\right]$, де $T_{11} \in \mathrm{GL}(m, \mathrm{R})$ і $T_{22} \in$ $\mathrm{GL}(n, \mathrm{R})$, така, що $T_{0} M_{1}=M_{2} T_{0}$. Якщо характеристичні многочлени матриць $A$ і $B$ взаємно прості, то наступна лема доводить дану гіпотезу для матриць над областю головних ідеалів.

Твердження 2.4. Нехай для матриць $A \in M_{m, m}(\mathrm{R}), B \in M_{n, n}(\mathrm{R})$, $C_{1}, C_{2} \in M_{n, m}(\mathrm{R})$ матрuиi

$$
M_{1}=\left[\begin{array}{cc}
A & 0_{m, n} \\
C_{1} & B
\end{array}\right] \quad m a \quad M_{2}=\left[\begin{array}{cc}
A & 0_{m, n} \\
C_{2} & B
\end{array}\right]
$$


подібні. Якщо характеристичні многочлени матрищь $A$ i $B$ взаємно прості, то існуе матриия $T_{0}=\left[\begin{array}{cc}T_{11} & 0_{m, n} \\ T_{21} & T_{22}\end{array}\right]$, де $T_{11} \in \mathrm{GL}(m, \mathrm{R}) i T_{22} \in$ $\mathrm{GL}(n, \mathrm{R}), \operatorname{ma\kappa a}, щ_{о} T_{0} M_{1}=M_{2} T_{0}$.

Доведення. Так як матриці $M_{1}$ і $M_{2}$ подібні, то існує матриця

$$
T_{0}=\left[\begin{array}{ll}
T_{11} & T_{12} \\
T_{21} & T_{22}
\end{array}\right] \in \mathrm{GL}(m+n, \mathrm{R}),
$$

де $T_{11} \in M_{m, m}(\mathrm{R})$ і $T_{22} \in M_{n, n}(\mathrm{R})$ така, що $T_{0} M_{1}=M_{2} T_{0}$. Виконавши множення в обох частинах цієї рівності отримуємо

$$
\left[\begin{array}{cc}
T_{11} A+T_{12} C_{1} & T_{12} B \\
T_{21} A+T_{22} C_{1} & T_{22} B
\end{array}\right]=\left[\begin{array}{cc}
A T_{11} & A T_{12} \\
C_{2} T_{11}+B T_{21} & C_{2} T_{12}+B T_{22}
\end{array}\right] .
$$

Із рівності $T_{12} B=A T_{12}$ отримуємо $T_{12}\left(I_{n} \lambda-B\right)=\left(I_{m} \lambda-A\right) T_{12}$. Так як характеристичні многочлени матриць $A$ і $B$ взаємно прості, то рівність $T_{12} B=A T_{12}$ справедлива лише у випадку, коли $T_{12}=0_{m, n}$. Отже, $T_{0}$ - нижня трикутна матриця, тобто $T_{12}=0_{m, n}$ i $T_{11} \in \mathrm{GL}(m, \mathrm{R})$ та $T_{22} \in \mathrm{GL}(n, \mathrm{R})$. Твердження доведено.

\section{3. МАТРИЦІ З НЕЗВІДНИМ МІНІМАЛЬНИМ КВАДРАТИЧНИМ МНОГОЧЛЕНОМ}

В цій частині опишемо структуру матриць з незвідними мінімальними многочленами $m(\lambda)=\lambda^{2}+m_{1} \lambda+m_{2} \in \mathrm{R}[\lambda]$ відносно перетворень подібності. Відомо, що матриця $A \in M_{n, n}(\mathrm{R})$ подібна нижній трикутній матриці (формі) Хессенберга $H=\left[h_{i j}\right]_{i, j=n}^{n}$, де $h_{i j}=0$ для всіх $j>i+1$, див. [12, Theorem III.1]. Нижче буде доведено, що для матриці $A$ з незвідним мінімальним многочленом $m(\lambda)$ нижня матриця Хессенберга є блочно-трикутною матрицею з блоками вимірності $2 \times 2$ на головній діагоналі та з характеристичними многочленами $m(\lambda)$.

Теорема 3.1. Нехай $A \in M_{n, n}(\mathrm{R})$ - матриия з незвідним мінімальним многочленом $m(\lambda)=\lambda^{2}+m_{1} \lambda+m_{2} \in \mathrm{R}[\lambda]$. Тодi:

а) існуе матриияя $T \in \mathrm{GL}(n, \mathrm{R})$ така, щ,

$$
T^{-1} A T=\left[\begin{array}{ccccc}
A_{11} & 0_{2,2} & \ldots & \ldots & 0_{2,2} \\
A_{21} & A_{22} & 0_{2,2} & \ldots & 0_{2,2} \\
\ldots & \ldots & \ldots & \ldots & \ldots \\
A_{k 1} & A_{k 2} & \ldots & A_{k, k-1} & A_{k k}
\end{array}\right],
$$

де $A_{i i} \in M_{2,2}(\mathrm{R})$ матриці з характеристичним многочленом $m(\lambda)$ для всіx $i=1,2, \ldots, k$;

б) $n=2 k$. 
Доведення. Якщо $n=2$, то твердження теореми очевидне. Доведемо, що в кільці $M_{3,3}(\mathrm{R})$ не існує матриць із незвідним мінімальним квадратичним многочленом. Припустимо, що існує матриця $A_{0} \in M_{3,3}(\mathrm{R})$ з мінімальним незвідним многочленом $m(\lambda)=\lambda^{2}+m_{1} \lambda+m_{2} \in \mathrm{R}[\lambda]$. Тоді їі характеристичний многочлен допускає зображення у вигляді добутку $a(\lambda)=\left(\lambda-\lambda_{1}\right) m(\lambda)$, де $\lambda_{1} \in \mathrm{R}$. На підставі твердження 2.2 та зауваження 2.1 отримуємо $m\left(\lambda_{1}\right)=0$, що неможливо, оскільки $m(\lambda) \Theta$ незвідним многочленом.

Нехай $n \geq 4$. Матрицю $A$ запишемо у вигляді

$$
A=\left[\begin{array}{ccccc}
a_{11} & a_{12} & a_{13} & \ldots & a_{1 n} \\
a_{21} & a_{22} & a_{23} & \ldots & a_{2 n} \\
\ldots & \ldots & \ldots & \ldots & \ldots \\
a_{n 1} & a_{n 2} & a_{n 3} & \ldots & a_{n n}
\end{array}\right]
$$

Так як $m(\lambda)$ є незвідним многочленом, то матриця $A$ перетвореннями подібності не може бути приведена до вигляду $\left[\begin{array}{cccc}\alpha & 0 & \ldots & 0 \\ A_{21} & & \bar{A}_{22}\end{array}\right]$, де $\bar{A}_{22} \in M_{n-1, n-1}(\mathrm{R})$. Очевидно, що $\left[\begin{array}{llll}a_{12} & a_{13} & \ldots & a_{1 n}\end{array}\right] \neq 0_{1, n-1}$. Матрицю $A$ перетвореннями подібності будемо приводити до нижньої матриці Хессенберга.

Нехай $b_{12} \in \mathrm{R}$ - найбільший спільний дільник цих елементів. Для рядка $\left[\begin{array}{llll}a_{12} & a_{13} & \ldots & a_{1 n}\end{array}\right] \in M_{1, n-1}(\mathrm{R})$ існує матриця $U_{1} \in \mathrm{GL}(n-1, \mathrm{R})$ (див. [12], Corollary II.1) така, що

$$
\left[\begin{array}{llll}
a_{12} & a_{13} & \ldots & a_{1 n}
\end{array}\right] U_{1}=\left[\begin{array}{llll}
b_{12} & 0 & \ldots & 0
\end{array}\right] .
$$

Отже, для матриці $T_{1}=\operatorname{diag}\left(e, U_{1}\right) \in \mathrm{GL}(n, \mathrm{R})$ отримуємо

$$
T_{1}^{-1} A T_{1}=A_{1}=\left[\begin{array}{cccccc}
a_{11} & b_{12} & 0 & \ldots & \ldots & 0 \\
\alpha_{21} & \alpha_{22} & \alpha_{23} & \alpha_{24} & \ldots & \alpha_{2 n} \\
\ldots & \ldots & \ldots & \ldots & \ldots & \ldots \\
\alpha_{n 1} & \alpha_{n 2} & \alpha_{n 3} & \alpha_{n 4} & \ldots & \alpha_{n n}
\end{array}\right] .
$$

Розглянемо елементи другого рядка $\left\{\alpha_{23}, \alpha_{24}, \ldots, \alpha_{2 n}\right\}$ матриці $A_{1}$. Якщо $\alpha_{23}=\alpha_{24}=\cdots=\alpha_{2 n}=0$, то матриця $A_{1}$ має вигляд

$$
A_{1}=\left[\begin{array}{cc}
A_{11} & 0_{2, n-2} \\
\bar{A}_{21} & \bar{A}_{22}
\end{array}\right],
$$

де $A_{11}=\left[\begin{array}{ll}a_{11} & b_{12} \\ \alpha_{21} & \alpha_{22}\end{array}\right]$ і $\bar{A}_{22} \in M_{n-2, n-2}(\mathrm{R})$. Згідно зауваження $2.1 m(\lambda)$ характеристичний многочлен матриці $A_{11}$ і $m\left(\bar{A}_{22}\right)=\mathrm{O}$.

Припустимо, що не всі елементи $\left\{\alpha_{23}, \alpha_{24}, \ldots, \alpha_{2 n}\right\}$ дорівнюють нулю. Нехай далі $b_{23} \in \mathrm{R}$ - найбільший спільний дільник цих елементів. Для 
рядка $\left[\begin{array}{llll}\alpha_{23} & \alpha_{24} & \ldots & \alpha_{2 n}\end{array}\right] \in M_{1, n-2}(\mathrm{R})$ існує матриця $U_{2} \in \mathrm{GL}(n-2, \mathrm{R})$ така, що $\left[\begin{array}{llll}\alpha_{23} & \alpha_{24} & \ldots & \alpha_{2 n}\end{array}\right] U_{2}=\left[\begin{array}{llll}b_{23} & 0 & \ldots & 0\end{array}\right]$.

Отже, для матриці $T_{2}=\operatorname{diag}\left(I_{2}, U_{2}\right) \in \mathrm{GL}(n, \mathrm{R})$ отримуємо

$$
T_{2}^{-1} A_{1} T_{2}=A_{2}=\left[\begin{array}{ll|llll}
a_{11} & b_{12} & 0 & \ldots & \ldots & 0 \\
\alpha_{21} & \alpha_{22} & b_{23} & 0 & \ldots & 0 \\
\hline \beta_{31} & \beta_{32} & \beta_{33} & \beta_{34} & \ldots & \beta_{3 n} \\
\ldots & \ldots & \ldots & \ldots & \ldots & \ldots \\
\beta_{n 1} & \beta_{n 2} & \beta_{n 3} & \beta_{n 4} & \ldots & \beta_{n n}
\end{array}\right] .
$$

Матрицю $\left(A_{2}\right)^{2}$ запишемо в розгорнутому вигляді, (виділивши при цьому перший, другий та останній рядки та перший, другий та третій стовпчики), тобто:

\begin{tabular}{|c|c|c|c|c|}
\hline $\begin{array}{c}a_{11}^{2}+ \\
\alpha_{21} b_{12} \\
\end{array}$ & $\begin{array}{c}a_{11} b_{12}+ \\
\alpha_{22} b_{12}\end{array}$ & $b_{12} b_{23}$ & 0 & . 0 \\
\hline $\begin{array}{c}\alpha_{21} a_{11}+ \\
\alpha_{21} \alpha_{22}+ \\
b_{23} \beta_{31} \\
\end{array}$ & $\begin{array}{c}\alpha_{21} b_{12}+ \\
\alpha_{21}^{2}+ \\
b_{23} \beta_{32} \\
\end{array}$ & $\begin{array}{l}\alpha_{22} b_{23} \\
\beta_{33} b_{23}\end{array}$ & $b_{23} \beta_{34}$ & $\ldots \quad b_{23} \beta_{3 n}$ \\
\hline$\ldots$ & $\ldots$ & $\ldots$ & $\ldots$ & $\ldots \quad \ldots$ \\
\hline $\begin{array}{c}\beta_{n 1} b_{12}+ \\
\beta_{n 2} \alpha_{22}+ \\
\sum_{i=3}^{n} \beta_{n i} \beta_{i 1}\end{array}$ & $\begin{array}{c}\beta_{n 1} a_{11}+ \\
\beta_{n 2} \alpha_{21}+ \\
\sum_{i=3}^{n} \beta_{n i} \beta_{i 2}\end{array}$ & $\begin{array}{c}\beta_{n 2} b_{23}+ \\
\sum_{i=3}^{n} \beta_{n i} \beta_{i n}\end{array}$ & $\sum_{i=3}^{n} \beta_{n i} \beta_{i 4}$ & $\cdots \sum_{i=3}^{n} \beta_{n i} \beta_{i 3}$ \\
\hline
\end{tabular}

Із рівності $m\left(A_{2}\right)=0_{n, n}$ випливає $b_{12} b_{23}=0$. Так як $b_{12} \neq 0$, то $b_{23}=0$. Отже, для матриці $U_{1}=T_{1} T_{2} \in \mathrm{GL}(n, \mathrm{R})$ здобуваємо

$$
A_{2}=U_{1}^{-1} A_{1} U_{1}\left[\begin{array}{cc|ccc}
a_{11} & b_{12} & 0 & \ldots & 0 \\
\alpha_{21} & \alpha_{22} & 0 & \ldots & 0 \\
\hline \beta_{31} & \beta_{32} & \beta_{33} & \ldots & \beta_{3 n} \\
\ldots & \ldots & \ldots & \ldots & \ldots \\
\beta_{n 1} & \beta_{n 2} & \beta_{n 3} & \ldots & \beta_{n n}
\end{array}\right]=\left[\begin{array}{cc}
A_{11} & 0_{2, n-2} \\
\bar{A}_{21} & \bar{A}_{22}
\end{array}\right],
$$

де $A_{11}=\left[\begin{array}{ll}a_{11} & b_{12} \\ \alpha_{21} & \alpha_{22}\end{array}\right] \in M_{22}(\mathrm{R})$ і $\bar{A}_{22} \in M_{n-2, n-2}(\mathrm{R})$. На підставі зауваження 2.1 отримуємо $m\left(A_{11}\right)=0_{2,2}$, тобто $m(\lambda) \in$ характеристичним многочленом матриці $A_{11}$.

Так як $n \geq 4$ і $m\left(\bar{A}_{22}\right)=\mathrm{O}$, то надалі матрицю $\bar{A}_{22}$ перетвореннями подібності будемо приводити до блочно-трикутного вигляду. Застосовуючи до матриці $\bar{A}_{22}$ міркування аналогічні тим, що були викладені 
вище, за скінченну кількість перетворень подібності матрицю $A$ приведемо до вигляду (3.1). Одночасно отримуємо, що $n=2 k$. Теорему доведено.

4. МАТРИЦІ $з$ МІНІМАЛЬНИМ МНОГОЧЛЕНОМ $m(\lambda)=(\lambda-\alpha)(\lambda-\beta)$,

$$
\alpha \neq \beta
$$

Метою цього розділу є опис структури матриць із кільця $M_{n, n}(\mathrm{R})$ відносно перетворень подібності з мінімальними многочленами

$$
m(\lambda)=(\lambda-\alpha)(\lambda-\beta)
$$

де $\alpha, \beta \in \mathrm{R}$ i $\alpha \neq \beta$. Для матриць другого порядку над кільцем цілих чисел ця задача досліджувалась в роботах $[25,2]$.

Теорема 4.1. Нехай $A \in M_{n, n}(\mathrm{R})$ - матриия з характеристичним многочленом $a(\lambda)=(\lambda-\alpha)^{k}(\lambda-\beta)^{n-k}$, де $\alpha, \beta \in \mathrm{R}, \alpha \neq \beta, 1 \leq k<n$. Якщо $m(\lambda)=(\lambda-\alpha)(\lambda-\beta)-$ мінімальний многочлен матриці $A$, то для $A$ існуе така матриця $T \in \mathrm{GL}(n, \mathrm{R}), щ_{о}$

$$
T A T^{-1}=A_{\Phi}=\left[\begin{array}{cc}
\alpha I_{k} & 0_{k, n-k} \\
\Phi & \beta I_{n-k}
\end{array}\right]
$$

$\partial e$

$$
\Phi=\left[\begin{array}{ccccc|c}
\phi_{1} & 0 & \ldots & \ldots & 0 & \\
0 & \phi_{2} & 0 & \ldots & 0 & \\
\vdots & \vdots & \ddots & \vdots & \vdots & 0_{l, k-l} \\
0 & \ldots & 0 & \phi_{l-1} & 0 & \\
0 & \ldots & \ldots & 0 & \phi_{l} & \\
\hline \multicolumn{7}{c}{0_{n-k-l, l}} & & 0_{n-k-l, k-l}
\end{array}\right] \in M_{n-k, k}(\mathrm{R})
$$

$\phi_{j} \neq 0(\bmod (\alpha-\beta))$ для всіх $1 \leq j \leq l i \phi_{i} \mid \phi_{i+1}$ (ділить) для всіх $i=1,2, \ldots, l-1$.

Доведення. Нехай $m(\lambda)=(\lambda-\alpha)(\lambda-\beta)-$ мінімальний многочлен матриці $A$. Якщо $A=0_{n, n}(\bmod (\alpha-\beta))$, то на підставі [22, теорема 2.1], матриця $A$ подібна діагональній матриці $\operatorname{diag}\left(\alpha I_{k}, \beta I_{n-k}\right)$.

Якщо ж $A \neq 0_{n, n}(\bmod (\alpha-\beta))$, то на підставі твердження 2.3 для $A$ існує матриця $T_{1} \in \mathrm{GL}(n, \mathrm{R})$ така, що

$$
T_{1} A T_{1}^{-1}=A_{1}=\left[\begin{array}{cc}
\alpha I_{k} & 0_{k, n-k} \\
A_{21} & \beta I_{n-k}
\end{array}\right]
$$


Очевидно, що $A_{21} \in M_{n-k, k}(\mathrm{R})$ - ненульова матриця. Для $A_{21}$ існують матриці $U_{1} \in \mathrm{GL}(n-k, \mathrm{R})$ i $V_{1} \in \mathrm{GL}(k, \mathrm{R})$ такі, що

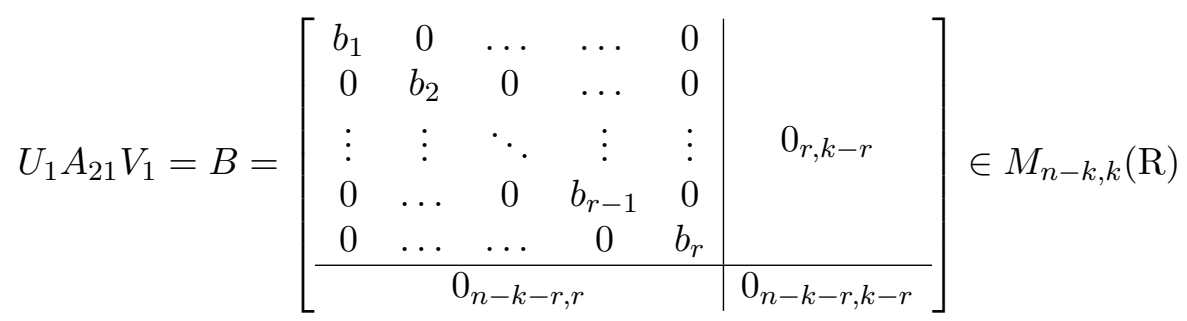

- форма Сміта матриці $A_{21}$, тобто $b_{j} \neq 0$ i $b_{i} \mid b_{i+1}$ (ділить) для всіх $i=1,2, \ldots, r-1$. Отже, для матриці $T_{2}=\operatorname{diag}\left(V_{1}^{-1}, U_{1}\right) \in \operatorname{GL}(n, \mathrm{R})$ отримуємо

$$
T_{2} A_{1} T_{2}^{-1}=A_{2}=\left[\begin{array}{cc}
\alpha I_{k} & \mathrm{O} \\
B & \beta I_{n-k}
\end{array}\right] .
$$

Серед елементів $b_{1}, b_{2}, \ldots, b_{r}$ виберемо ті, для яких $b_{i}=(\alpha-\beta) p_{i}$, де $p_{i} \in \mathrm{R}$. Якщо ж для деякого $1 \leq i_{0} \leq r$ виконується

$$
b_{i} \neq 0 \quad(\bmod (\alpha-\beta)),
$$

то покладемо $p_{i_{0}}=0$. За заданими елементами $\left\{p_{1}, p_{2}, \ldots, p_{r}\right\}$ побудуємо матрицю

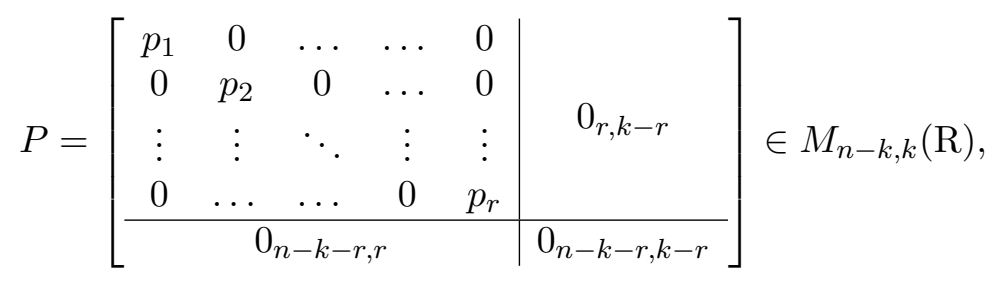

Легко перевірити, що для матриці $T_{3}=\left[\begin{array}{cc}I_{k} & 0_{n-k, k} \\ -P & I_{n-k}\end{array}\right] \in \mathrm{GL}(n, \mathrm{R})$ виконується наступна рівність

$$
T_{3} A_{2} T_{3}^{-1}=A_{2}=\left[\begin{array}{cc}
\alpha I_{k} & \mathrm{O} \\
C & \beta I_{n-k}
\end{array}\right]
$$

де

$$
C=\left[\begin{array}{ccccc|c}
c_{1} & 0 & \ldots & \ldots & 0 & \\
0 & c_{2} & 0 & \ldots & 0 & \\
\vdots & \vdots & \ddots & \vdots & \vdots & 0_{r, k-r} \\
0 & \ldots & 0 & c_{r-1} & 0 & \\
0 & \ldots & \ldots & 0 & c_{r} & \\
\hline \multicolumn{7}{c}{0_{n-k-r, r}} & 0_{n-k-r, k-r}
\end{array}\right] \in M_{n-k, k}(\mathrm{R}),
$$

причому $c_{i}=0$, якщо $b_{i}=(\alpha-\beta) p_{i}$ i $c_{i}=b_{i}$, якщо $b_{i} \neq 0(\bmod (\alpha-\beta))$. 
Оскільки $C$ діагональна матриця, то існують матриці перестановок $P_{l} \in \mathrm{GL}(n-k, \mathrm{R})$ і $P_{r} \in \mathrm{GL}(k, \mathrm{R})$ такі, що

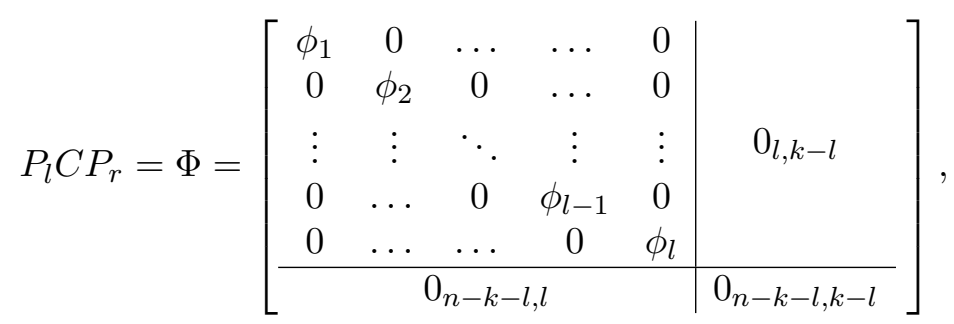

де $\phi_{j} \neq 0(\bmod (\alpha-\beta))$ для всіх $j=1, \ldots, l$. Очевидно, що $l \leq r$ i $\phi_{i} \mid \phi_{i+1}$ (ділить) для всіх $i=1, \ldots, l-1$.

Отже, для матриці $T_{4}=\operatorname{diag}\left(P_{r}^{-1}, P_{l}\right) \in \mathrm{GL}(n, \mathrm{R})$ отримуємо

$$
T_{4} A_{3} T_{4}^{-1}=A_{\Phi}=\left[\begin{array}{cc}
\alpha I_{k} & 0_{k, n-k} \\
\Phi & \beta I_{n-k}
\end{array}\right]
$$

Теорему доведено.

Нехай $\mathrm{R}=\mathbb{Z}$. Розглянемо $2 \times 2$ матриці $A=\left[\begin{array}{ll}1 & 0 \\ 2 & 6\end{array}\right]$ i $B=\left[\begin{array}{ll}1 & 0 \\ 3 & 6\end{array}\right]$ над $\mathbb{Z}$. Для матриці $W=\left[\begin{array}{cc}-1 & 0 \\ 1 & 1\end{array}\right] \in \mathrm{GL}(2, \mathbb{Z})$ виконується рівність $W A W^{-1}=B$. Отже, за умов теореми 4.1 матриця $\Phi$ визначена неоднозначно. Нижче вкажемо зв'язок між трикутними формами, які встановлені теоремою 4.1, для матриці $A \in M_{n, n}(\mathrm{R})$ з мінімальним многочленом $m(\lambda)=(\lambda-\alpha)(\lambda-\beta), \alpha \neq \beta$. Зауважимо, що наведений приклад не узгоджується з [2, теорема 5.2].

Припустимо, що за умов теореми 4.1 для $A \in M_{n, n}(\mathrm{R})$ існує матриця $V \in \mathrm{GL}(n, \mathrm{R})$ така, що $V A V^{-1}=A_{\Psi}=\left[\begin{array}{cc}\alpha I_{k} & 0_{k, n-k} \\ \Psi & \beta I_{n-k}\end{array}\right]$, де

$$
\Psi=\left[\begin{array}{ccccc|c}
\psi_{1} & 0 & \ldots & \ldots & 0 & \\
0 & \psi_{2} & 0 & \ldots & 0 & \\
\vdots & \vdots & \ddots & \vdots & \vdots & 0_{l, k-l} \\
0 & \ldots & 0 & \psi_{l-1} & 0 & \\
0 & \ldots & \ldots & 0 & \psi_{l} & \\
\hline \multicolumn{7}{c}{0_{n-k-l, l}} & & 0_{n-k-l, k-l}
\end{array}\right] \in M_{n-k, k}(\mathrm{R})
$$

$\psi_{j} \neq 0(\bmod (\alpha-\beta)), \psi_{i} \mid \phi_{i+1}$ для всіх $1 \leq i \leq l-1$ і $\Psi \neq \Phi$. Очевидно, що матриці $A_{\Phi}$ і $A_{\Psi}$ подібні, тобто

$$
W\left[\begin{array}{cc}
\alpha I_{k} & 0_{k, n-k} \\
\Phi & \beta I_{n-k}
\end{array}\right]=\left[\begin{array}{cc}
\alpha I_{k} & 0_{k, n-k} \\
\Psi & \beta I_{n-k}
\end{array}\right] W
$$


де $W \in \mathrm{GL}(n, \mathrm{R})$. Згідно твердження 2.4 матриця $W$ має вигляд

$$
W=\left[\begin{array}{cc}
W_{1} & 0_{k, n-k} \\
W_{21} & W_{2}
\end{array}\right] \text {, де } W_{1} \in G(k, \mathrm{R}) \text { i } W_{2} \in G(n-k, \mathrm{R}) .
$$

Крім цього, із рівності (4.1) отримуємо $W_{2} \Phi-\Psi W_{1}=(\beta-\alpha) W_{21}$. Ця рівність описує зв'язок між трикутними формами $A_{\Phi}$ і $A_{\Psi}$, які встановлені теоремою 4.1 для матриці $A \in M_{n, n}(\mathrm{R})$ з мінімальним многочленом $m(\lambda)=(\lambda-\alpha)(\lambda-\beta), \alpha \neq \beta$.

Нагадаємо, що матриця $A \in M_{n, n}(\mathrm{R})$ називається інволютивною, якщо $A^{2}=I_{n}$. Теорема 4.1 дозволила описати структуру інволютивних матриц над кільцем цілих чисел відносно перетворення подібності.

Наслідок 4.2. Нехай $a(\lambda)=(\lambda-1)^{k}(\lambda+1)^{n-k}-$ характеристичний многочлен інволютивної матриці $A \in M_{n, n}(\mathbb{Z})$, де $1 \leq k<n$. Тодi існуе $T \in \mathrm{GL}(n, \mathbb{Z})$ така, що

$$
T A T^{-1}=A_{r}=\left[\begin{array}{c|c}
I_{k} & 0_{k, n-k} \\
\hline J_{r} & -I_{n-k}
\end{array}\right],
$$

де $J_{r}=\left[\begin{array}{cc}I_{r} & 0_{k-r} \\ 0_{n-k-r, r} & 0_{n-k-r, k-r}\end{array}\right] \in M_{n-k, k}(\mathbb{Z})$. Число $r$ дорівнюе кількості інваріантних множників, які збігаються з одинищею, матриці $A+I_{n}$. При ивому матриця $A_{r}$ для $A$ визначена однозначно.

Доведення. Згідно теореми 4.1 для інволютивної матриці $A$ існує матриця $T \in \mathrm{GL}(n, \mathbb{Z})$ така, що $T A T^{-1}=A_{r}=\left[\begin{array}{c|c}I_{k} & 0_{k, n-k} \\ \hline J_{r} & -I_{n-k}\end{array}\right]$, де $J_{r}=\left[\begin{array}{cc}I_{r} & 0_{k-r} \\ 0_{n-k-r, r} & 0_{n-k-r, k-r}\end{array}\right] \in M_{n-k, k}(\mathbb{Z})$.

Припустимо, що для $A$ існує матриця $W \in \mathrm{GL}(, \mathbb{Z})$ така, що $W \neq T$ i

$$
W A W^{-1}=A_{l}=\left[\begin{array}{c|c}
I_{k} & 0_{k, n-k} \\
\hline J_{l} & -I_{n-k}
\end{array}\right], \quad \text { де } \quad J_{l}=\left[\begin{array}{cc}
I_{l} & 0_{k-l} \\
0_{n-k-l, l} & 0_{n-k-l, k-l}
\end{array}\right],
$$

$l \leq \min \{k, n-k\}$ i $l \neq r$, тобто $A_{l} \neq A_{r}$. Так як матриці $A_{l}$ і $A_{r}$ подібні, то матриці $\left(I_{n}+A_{l}\right)$ і $\left(I_{n}+A_{r}\right)$ теж подібні. Очевидно, якщо дві матриці подібні, то вони еквівалентні. Легко переконатись в тому, що $S_{l}=\operatorname{diag}\left(I_{l}, 2 I_{k-l}, 0, \ldots, 0\right)$ i $S_{r}=\operatorname{diag}\left(I_{r}, 2 I_{k-r}, 0, \ldots, 0\right)-$ форми Сміта матриць $\left(I_{n}+A_{l}\right)$ і $\left(I_{n}+A_{r}\right)$ відповідно. Так як $l \neq r$, то форми Сміта $S_{l}$ і $S_{l}$ не еквівалентні. Отже, припущення про те, що $l \neq r$ є невірним. Тим самим доведено єдиність матриці $A_{r}$ для інволютивної матриці $A$. Число $r$ дорівнює кількості інваріантних множників, які збігаються 3 одиницею 1 , форми Сміта матриці $I_{n}+A_{r}$. Наслідок доведено. 
Із теореми 4.1 та роботи [24] отримуємо описання структури матриць другого порядку над областю головних ідеалів із звідним характеристичним многочленом відносно перетворення подібності.

Наслідок 4.3. Нехай $A \in M_{2,2}(\mathrm{R})$ - матриия з характеристичним многочленом $a(\lambda)=(\lambda-\alpha)(\lambda-\beta), \quad$ де $\alpha, \beta \in \mathrm{R}$. Тодi A подібна одній із матричь:

а) $A_{1}=\left[\begin{array}{ll}\alpha & 0 \\ 0 & \beta\end{array}\right]$, якщо $\alpha \neq \beta i A=0_{2,2}(\bmod (\alpha-\beta))$.

б) $A_{2}=\left[\begin{array}{ll}\alpha & 0 \\ r & \beta\end{array}\right]$, якщо $\alpha \neq \beta i A \neq 0_{2,2}(\bmod (\alpha-\beta))$, а елемент $r \in \mathrm{R}$ належить класу лишків за модулем ідеалу $(\alpha-\beta) \mathrm{R}$. Якщо ж матриия $A$ подібна матрииі $\widetilde{A}_{2}=\left[\begin{array}{ll}\alpha & 0 \\ \widetilde{r} & \beta\end{array}\right]$, де елемент $\widetilde{r}$ теж належить класу лишків за модулем ідеалу $(\alpha-\beta) \mathrm{R}$, то існуе оборотній елемент $u \in \mathrm{R}$ такий, що $\widetilde{r}-r u=0(\bmod (\alpha-\beta))$.

в) $A_{3}=\left[\begin{array}{ll}\alpha & 0 \\ b & \alpha\end{array}\right]$, якщо $\alpha=\beta$. Елемент $b \in \mathrm{R}$ визначений однозначно з точністю до асоиійованості.

Зауважимо, що умова б) наслідку 4.3 уточнює [2, теорема 5.2].

Автор висловлює щиру вдячність рецензенту за увагу до даної статті та слушні зауваження і поради.

\section{ЛiтературА}

[1] N. Avni, U. Onn, A. Prasad, L. Vaserstein. Similarity classes of $3 \times 3$ matrices over a local principal ideal ring. Communications Algebra, 37(8):2601-2615, 2009.

[2] A. Behn, A. B. Van der Merwe. An algorithmic version of the theorem by Latimer and MacDuffee for 2×2 integral matrices. Linear Algebra Appl., 346(1-3):1-14, 2002.

[3] W. C. Brown. Matrices over commutative rings, volume 169 of Pure and Applied Mathematics. New York: Marcel Dekker, 1993.

[4] J. T. Campbell, E. C. Trouy. When are two elements of GL(2, Z) similar? Linear Algebra Appl., 157:175-184, 1991.

[5] J. D. Dixon. An isomorphism criterion for modules over a principal ideal domain. Linear Multilin. Algebra, 8(1):69-72, 1979.

[6] Sh. Friedland. Matrices: Algebra, Analysis and Applications. World Scientific, 2015.

[7] R. M. Guralnick. Similarity of matrices over commutative rings. Linear Algebra Appl., 157:55-68, 1991.

[8] W. H. Gustafson. Roth's theorems over commutative rings. Linear Algebra Appl., 23:245-251, 1979.

[9] W. H. Gustafson. On matrix similarity over commutative rings. Linear Multilin. Algebra, 10(3):249-252, 1981.

[10] C. G. Latimer, C. C. MacDuffee. A correspondence between classes of ideals and classes of matrices. Annals of Math. Second Series., 34(2):313-316, 1933. 
[11] B. R. McDonald. Similarity of matrices over artinian principal ideal rings. Linear Algebra Appl., 21(2):153-162, 1978.

[12] M. Newman. Integer Matrices. New York: Acad.Press, 1972.

[13] O. Taussky. Matrices of rational integers. Bull. Amer. Math. Soc., 66(5):327-345, 1960.

[14] H. K. Wimmer. Problem 45-7, A generalization of Roth's theorem. Bull. Internat. Linear Algebra Society, 45:48, 2010.

[15] В. М. Бондаренко. О подобии матриц над кольцами классов вычетов. Математический сборник. К.: Наукова думка, 1:275-277, 1976.

[16] Ф. Р. Гантмахер. Теория матрии. М.: Наука, 1978.

[17] Ф. Груневальд, Н. К. Иыуду. Задача сопряжения для $2 \times 2$-матриц над кольцами многочленов. Современная математика и ее прилож., 30(3):31-45, 2005.

[18] П. М. Гудивок. О модулярных и целочисельных представлениях конечных групп. ДАН СССР, 214(5):993-996, 1974.

[19] Ю. А. Дрозд. О ручных и диких матричных задачах. Матричные задачи, 1:104114, 1977.

[20] Ю. А. Дрозд. Ручные и дикие матричные задачи. Представления и квадратичные бормы, 1:39-74, 1979.

[21] А. А. Нечаев. О подобии матриц над коммутативным локальным артиновым кольцом. Труды сем. им. И. Г. Петровского, 9:81-101, 1989.

[22] В. М. Прокіп. Діагоналізованість матриць над областю головних ідеалів з мінімальним многочленом $m(\lambda)=(\lambda-\alpha)(\lambda-\beta),(\alpha \neq \beta)$. Укр. мат. вісник, $7(2): 212$ $219,2010$.

[23] В. М. Прокіп. Діагоналізовність матриць над областю головних ідеалів. Укр. мат. журнал, 64(2):283-288, 2012.

[24] В. М. Прокіп. Структура матриць рангу один над областю головних ідеалів відносно перетворення подібності. Мат. методи та фiз.-мех. поля, 59(3):68-76, 2017.

[25] С. В. Сидоров, В. Н. Шевченко. О подобии матриц второго порядка над кольцом целых чисел. Изв. вузов. Математика, 4:57-64, 2006.

[26] Д. А. Супруненко. О сопряженности матриц над кольцом вычетов. ДАН БССР, 8(11):693-695, 1964.

Надійшла до редакиії 20 грудня 2018, прийнята до друку 3 травня 2019.

Володимир Прокіп

ІППММ НАН УКРАЇНИ, НАУКОВА ЗБ, ЛЬВІв, УКРАїНА, 79060

Email: v.prokip@gmail.com 